Supporting Information for:

\title{
Chain Exchange Kinetics of Bottlebrush Block
}

\section{Copolymer Micelles}

\author{
Seyoung Kim ${ }^{1,2}$, Sangho Lee ${ }^{2}$, Soo-Hyung Choi ${ }^{2 *}$, and Kookheon Char ${ }^{1,3 *}$ \\ ${ }^{1}$ Department of Chemical and Biological Engineering, Seoul National University, Seoul \\ 08826, Republic of Korea \\ ${ }^{2}$ Department of Chemical Engineering, Hongik University, Seoul 04066, Republic of Korea \\ ${ }^{3}$ The National Creative Research Initiative Center for Intelligent Hybrids, Seoul National \\ University, Seoul 08826, Republic of Korea
}

*Authors for correspondence : (S. C.) shchoi@hongik.ac.kr, (K. C.) khchar@snu.ac.kr 


\section{Synthesis and Characterization}

(a)
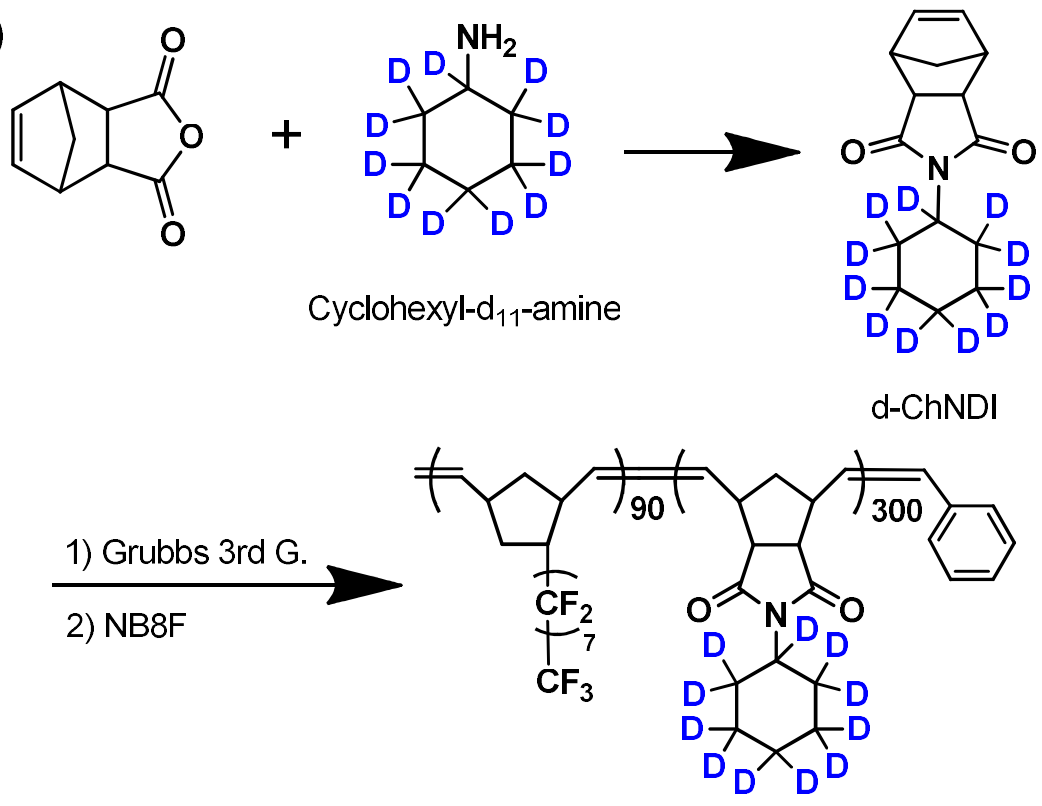

PF-block-dPC

(b)

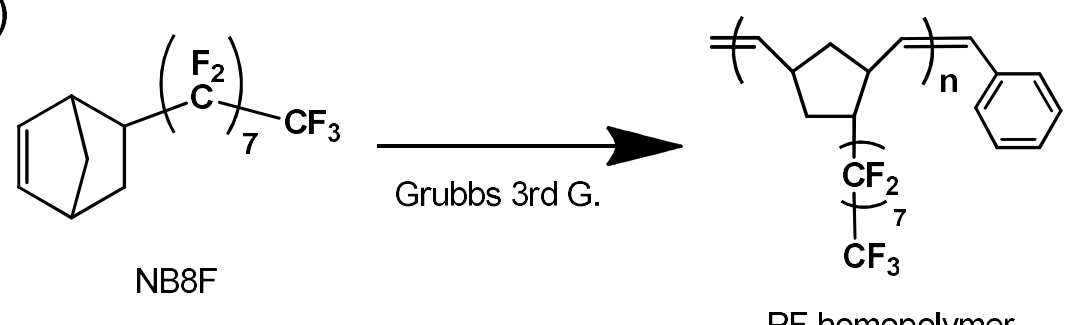

PF homopolymer

Figure S1. Representative synthetic scheme of (a) PF-b-dPC diblock copolymer and (b) PF homopolymer. As a counterpart, PF- $b$-hPC was synthesized with hydrogenated cyclohexylamine. All block copolymers and homopolymers have phenyl end groups derived from Grubbs 3rd generation catalyst, although the end group was only displayed in PF product for simplicity. 

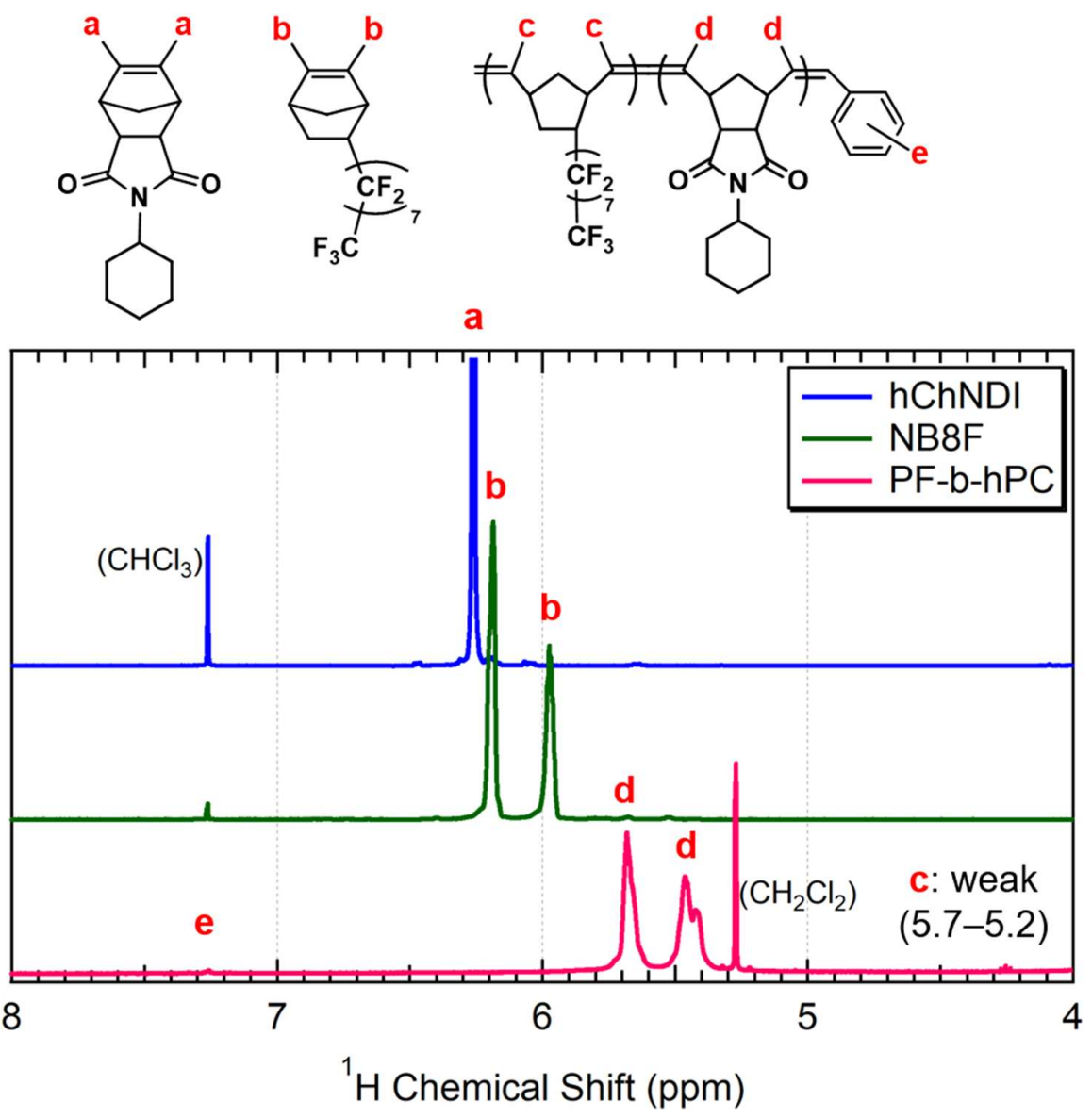

Figure S2. ${ }^{1} \mathrm{H}$ NMR spectra of the monomers (i.e., hChNDI and $\mathrm{NB} 8 \mathrm{~F}$ ) in $\mathrm{CDCl}_{3}$ and the PF$b$-hPC block copolymer in $\mathrm{CD}_{2} \mathrm{Cl}_{2}$ at $25{ }^{\circ} \mathrm{C}$. The peaks from norbornenyl double bonds (Ha and $\mathrm{Hb}, 6.3-5.9 \mathrm{ppm})$ are extinct for $\mathrm{PF}-b$-hPC, indicating that the monomers were nearly completely consumed (conversion $>99.5 \%$ ) during the ring-opening metathesis polymerization (ROMP). The splitting in $\mathrm{Hb}$ and $\mathrm{Hd}$ arises from the fluoroalkyl graftinginduced asymmetry and the cis/trans isomers, respectively. The Hc peak was weak and broad since PF block segregates from the $\mathrm{CD}_{2} \mathrm{Cl}_{2}$ solvent. A small He peak from the chain end phenyl groups was also discernible. The solvent peaks are denoted. 


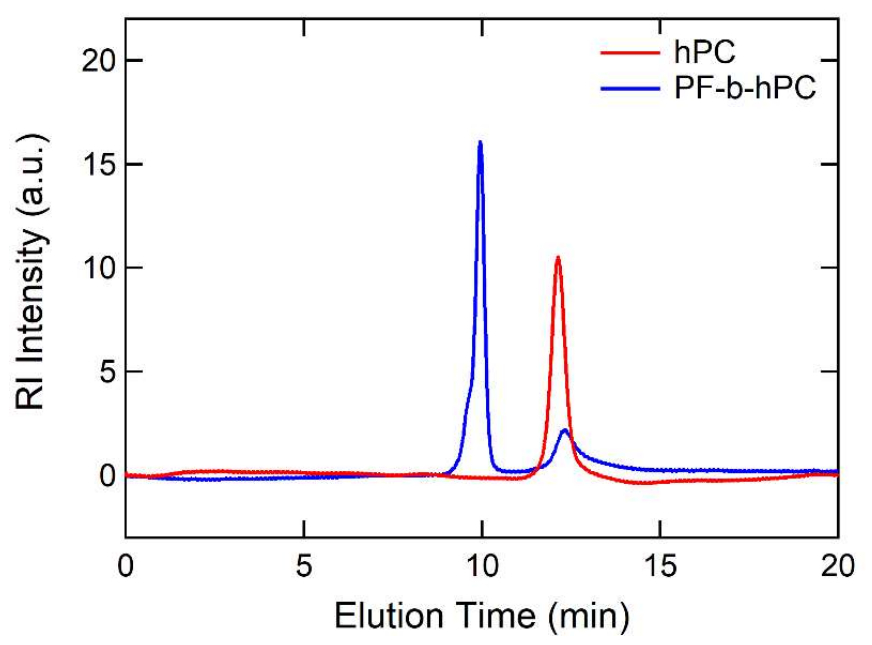

Figure S3. Representative SEC traces for hPC $\left(N_{\mathrm{PC}}=300\right.$, red $)$ and PF- $b-\mathrm{hPC}\left(N_{\mathrm{PF}}=85\right.$ and $N_{\mathrm{PC}}=300$, blue). Due to the limited solubility of PF block in THF, an eluent, a strong peak near 10 min for PF- $b$-hPC is associated with micellar aggregates, and a weak peak near 12 min with singly dispersed chains. The apparent overlap between the singly dispersed proportion of $\mathrm{PF}-b$-hPC and the hPC is attributed to the poor solubility of the PF block in THF, and thus the reduced hydrodynamic volume for PF- $b$-hPC. This is consistent with previous reports of molecular weight characterization for fluorinated copolymers. ${ }^{1-3}$ It is noted that the PF- $b$-hPC sample does not contain hPC residue because hPC homopolymers were selectively removed after polymerization. 


\section{Differential Scanning Calorimetry (DSC)}

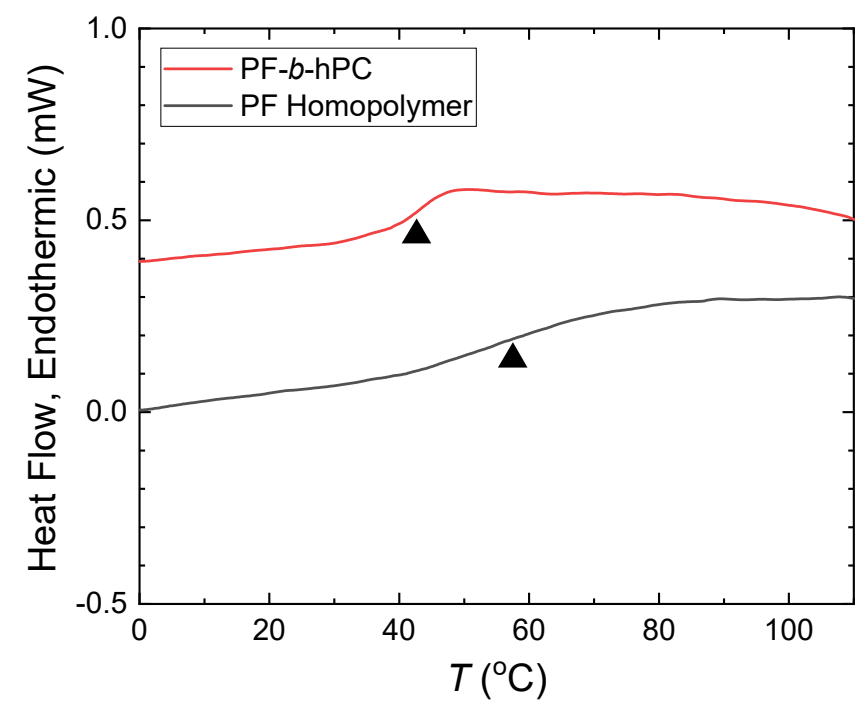

Figure S4. DSC curves from the second heating of PF homopolymer $(M=15 \mathrm{~kg} / \mathrm{mol})$ and PF$b$-hPC diblock copolymer used in the present study. Curves were vertically shifted for clarity. The mid-points of glass transitions $\left(T_{\mathrm{g}}\right)$ were identified as triangles.

Table S1. Neutron SLD and gravimetric density.

\begin{tabular}{ccc}
\hline Component & SLD $\left[10^{-6} \AA^{-2}\right]$ & $\rho[\mathrm{g} / \mathrm{ml}]$ \\
\hline PF & 3.43 & 1.80 \\
$\mathrm{hPC}$ & 1.56 & 1.28 \\
$\mathrm{dPC}$ & $4.58^{\mathrm{a}}$ & $1.33^{\mathrm{b}}$ \\
$\mathrm{hTHF}$ & 0.18 & 0.89 \\
$\mathrm{dTHF}$ & 6.35 & 0.99 \\
\hline
\end{tabular}

${ }^{\mathrm{a}}$ Neutron SLD of dPC was estimated using the density of $\mathrm{dPC}$ and the characterized deuterium substitution of 9.5 hydrogens per repeat unit (from ${ }^{1} \mathrm{H}$ NMR analysis). ${ }^{b}$ The density of dPC is calculated assuming the same molecular volume of dPC with that of $\mathrm{hPC}$. 


\section{Formalism of TR-SANS scattering model}

For the given set of scattering length densities (SLDs) and structural parameters of micelles, the extent of chain exchange can be evaluated through the relaxation function, $R(t)$. We introduce the exact formalism of $R(t)$ and corollaries for the isotopic micelle mixtures in an isotopic solvent mixture. The scattering model is based on Pedersen et al. ${ }^{4}$ who described the micelle as a combination of spherical core and Gaussian corona chains grafted at the surface of the core. The scattering intensity of individual micellar solutions $\left(I_{i}\right.$, where $i$ denotes either hydrogenated $(\mathrm{H})$ or deuterated (D) version of PF- $b$-PC block copolymers) is expressed as

$$
\begin{aligned}
I_{i} & =\left(N_{\mathrm{p}, i} / V\right)\left\{P_{\mathrm{mic}, i}(q)+[S(q)-1] A_{\mathrm{mic}, i}(q)^{2}\right\} \\
& =V^{-1}\left\{N_{\mathrm{p}, i} P_{\mathrm{mic}, i}(q)+\left[(S(q)-1) / N_{\mathrm{p}, i}\right]\left[N_{\mathrm{p}, i} A_{\mathrm{mic}, i}(q)\right]^{2}\right\},
\end{aligned}
$$

where $N_{\mathrm{p}, i}$ is the number of micelles, $V$ is the volume of the system, $P_{\mathrm{mic}, i}(q)$ is the scattering form factor and $A_{\text {mic, } i}(q)$ is the form factor amplitude of the radial scattering length distribution of micelle. $S(q)$ is the micelle structure factor, and in this study the Percus-Yevick hard-sphere structure factor was assumed. $P_{\text {mic }}(q)$ and $A_{\text {mic }}(q)$ are expanded below for further derivation:

$$
\begin{aligned}
P_{\text {mic }}(q)= & {\left[N_{\text {agg }} \beta_{\text {core }} A_{\text {core }}(q)\right]^{2}+2 N_{\text {agg }}^{2} \beta_{\text {core }} A_{\text {core }}(q) \beta_{\text {corona }} A_{\text {corona }}(q) } \\
& +N_{\text {agg }}\left(N_{\text {agg }}-1\right)\left[\beta_{\text {corona }} A_{\text {corona }}(q)\right]^{2}+N_{\text {agg }} \beta_{\text {corona }} P_{\text {corona }}(q) \\
A_{\text {mic }}(q)= & N_{\text {agg }}\left[\beta_{\text {core }} A_{\text {core }}(q)+\beta_{\text {corona }} A_{\text {corona }}(q)\right]
\end{aligned}
$$

where $N_{\text {agg }}$ is the aggregation number, $\beta_{j}=v_{j}\left(\rho_{j}-\rho_{\text {solvent }}\right)$ is the scattering length contrast $(j$ denotes either core or corona), $v_{j}$ is the molecular volume of the chain and $\rho_{j}$ is the scattering length density (SLD). $A_{\text {core }}(q)$ and $A_{\text {corona }}(q)$ are the normalized scattering amplitude of the core and the corona, respectively, and $P_{\text {corona }}(q)$ is the form factor of single corona chain. ${ }^{5}$

Then, we adopted ideas of the mixing rule from Choi et al. ${ }^{6}$ to treat the binary mixture of isotopic micelles. In the absence of chain exchange, binary mixture solutions of PF- $b$-hPC and 
PF- $b$-dPC micelles with identical structure (namely, $N_{\text {agg }}$ and the form factors $A_{\text {core }}(q), A_{\text {corona }}(q)$, and $\left.P_{\text {corona }}(q)\right)$ and interparticle interactions $(S(q))$ could be developed in a similar fashion with eq S1,

$$
I_{\mathrm{mix}}=V^{-1}\left\{N_{\mathrm{p}, \mathrm{H}} P_{\mathrm{mic}, \mathrm{H}}(q)+N_{\mathrm{p}, \mathrm{D}} P_{\mathrm{mic}, \mathrm{D}}(q)+\left[(S(q)-1) / N_{\mathrm{p}}\right]\left[N_{\mathrm{p}, \mathrm{H}} A_{\mathrm{mic}, \mathrm{H}}(q)+N_{\mathrm{p}, \mathrm{D}} A_{\mathrm{mic}, \mathrm{D}}(q)\right]^{2}\right\},
$$

where $N_{\mathrm{p}}=N_{\mathrm{p}, \mathrm{H}}+N_{\mathrm{p}, \mathrm{D}}$. Fortunately, this complicated expression could be simplified by the following conditions. First, if the numbers of PF- $b$-hPC and PF- $b$-dPC micelles are equal $\left(N_{\mathrm{p}, \mathrm{H}}\right.$ $\left.=N_{\mathrm{p}, \mathrm{D}} \equiv N_{\mathrm{p}} / 2\right)$, then eq $\mathrm{S} 3$ is reduced to

$$
\begin{aligned}
& I_{\text {mix }}=V^{-1}\left\{\left(N_{\mathrm{p}} / 2\right)\left[P_{\text {mic }, \mathrm{H}}(q)+P_{\text {mic }, \mathrm{D}}(q)\right]+\left[(S(q)-1) / N_{\mathrm{p}}\right]\left(N_{\mathrm{p}}^{2} / 4\right)\left[A_{\mathrm{mic}, \mathrm{H}}(q)+A_{\text {mic }, \mathrm{D}}(q)\right]^{2}\right\} \\
& =\left(N_{\mathrm{p}} / V\right)\left\{\left[P_{\mathrm{mic}, \mathrm{H}}(q)+P_{\mathrm{mic}, \mathrm{D}}(q)\right] / 2+(S(q)-1)\left[\left(A_{\text {mic }, \mathrm{H}}(q)+A_{\text {mic }, \mathrm{D}}(q)\right) / 2\right]^{2}\right\} .
\end{aligned}
$$

Second, if the SLD of solvent is matched to the average of hPC and $\mathrm{dPC}$ (i.e., zero-averagecontrast matching), then $\beta_{\mathrm{hPC}}=-\beta_{\mathrm{dPC}} \equiv \beta_{\text {corona. Therefore, }}$

$$
\begin{aligned}
P_{\text {mic }, \mathrm{H}}(q)+P_{\text {mic }, \mathrm{D}}(q)= & 2\left[N_{\mathrm{agg}} \beta_{\text {core }} A_{\text {core }}(q)\right]^{2}+2 N_{\mathrm{agg}}\left(N_{\mathrm{agg}}-1\right)\left[\beta_{\text {corona }} A_{\text {corona }}(q)\right]^{2} \\
& +2 N_{\mathrm{agg}} \beta_{\text {corona }}^{2} P_{\text {corona }}(q), \\
A_{\text {mic }, \mathrm{H}}(q)+A_{\text {mic }, \mathrm{D}}(q)= & N_{\mathrm{agg}}\left[\beta_{\text {corona }} A_{\text {corona }}(q)+\beta_{\text {corona }} A_{\text {corona }}(q)\right]+2 N_{\mathrm{agg}} \beta_{\text {core }} A_{\text {core }}(q) .
\end{aligned}
$$

Then, eq S4 is further reduced to

$$
I_{\text {mix }}=\left(N_{\mathrm{p}} / V\right)\left\{\left[N_{\mathrm{agg}} \beta_{\text {core }} A_{\text {core }}(q)\right]^{2} S(q)+N_{\text {agg }}\left(N_{\text {agg }}-1\right)\left[\beta_{\text {corona }} A_{\text {corona }}(q)\right]^{2}\right\}+\mathrm{C}(q),
$$

where $\mathrm{C}(q) \equiv\left(N_{\mathrm{p}} / V\right) N_{\mathrm{agg}} \beta_{\text {corona }}{ }^{2} P_{\text {corona }}(q)$ denotes the self-scattering from the individual corona chains.

Indeed, eq S5 is not only capable of describing the mixture without exchange, but also the mixture where some exchange has been undergone. If the chains are exchanged, the mean scattering length contrast between the "mixed" corona and the solvent, $\left|\beta_{\text {mix }}(t)\right|$, would decrease 
as a function of elapsed time $t$ after mixing. ${ }^{7}$ The only time-dependent term from eq S5 is then $\beta_{\text {mix }}(t)$, hence the time-dependent SANS intensity is

$$
I_{\text {mix }}(t)=\left(N_{\mathrm{p}} / V\right)\left\{\left[N_{\mathrm{agg}} \beta_{\text {core }} A_{\text {core }}(q)\right]^{2} S(q)+N_{\text {agg }}\left(N_{\text {agg }}-1\right)\left[\beta_{\text {mix }}(t) A_{\text {corona }}(q)\right]^{2}\right\}+\mathrm{C}(q),
$$

where the self-scattering term, $\mathrm{C}(q)=\left(N_{\mathrm{p}} / V\right) N_{\mathrm{agg}} \beta_{\text {corona }}^{2} P_{\text {corona }}(q)$, is independent of $t$.

The followings are experimentally important corollaries, from which the final expression of chain exchange, $R(t)$, is constructed,

$$
\begin{aligned}
& I_{\infty}=I_{\text {mix }}(t \rightarrow \infty)=\left(N_{\mathrm{p}} / V\right) N_{\mathrm{agg}}{ }^{2}\left[\beta_{\text {core }} A_{\text {core }}(q)\right]^{2} S(q)+\mathrm{C}(q) \\
& I_{0}=I_{\text {mix }}(t=0) \approx\left(N_{\mathrm{p}} / V\right) N_{\mathrm{agg}}{ }^{2}\left\{\left[\beta_{\text {core }} A_{\text {core }}(q)\right]^{2} S(q)+\left[\beta_{\text {mix }, 0} A_{\text {corona }}(q)\right]^{2}\right\}+\mathrm{C}(q) \\
& R(t)=\left\{\left[I_{\text {mix }}(t)-I_{\infty}\right] /\left[I_{0}-I_{\infty}\right]\right\}^{1 / 2}=\beta_{\text {mix }}(t) / \beta_{\text {mix }, 0},
\end{aligned}
$$

where $\beta_{\text {mix }, 0}=\beta_{\text {mix }}(0)$. In conclusion, eq S9 suggests that $R(t)$, described at eq 1 of the main text, is the normalized contrast of the corona in PF- $b$-PC micelles.

For evaluation of $I_{0}(q)$ in the main text, eq S8 was used with parameters such as $N_{\text {agg }}$ and $A_{\text {corona }}(q)$ obtained from the model fitting of individual micelles. $I_{0}(q)$ at several temperatures were compared with the SANS measurements obtained from the initial beam exposure of the isotopic mixture (Fig. S4). At $30{ }^{\circ} \mathrm{C}$, the chain exchange during exposure time of 5 min was minimal, and the simulated $I_{0}(q)$ curve overlaps with the experimental curve, which confirms that our derivation using eq S8 agrees with the SANS experiments. However, at higher temperature, the experimental curves systematically deviate from the simulated $I_{0}(q)$, which is attributed to faster chain exchange. Therefore, the simulated $I_{0}(q)$ was employed to calculate $R(t)$ 

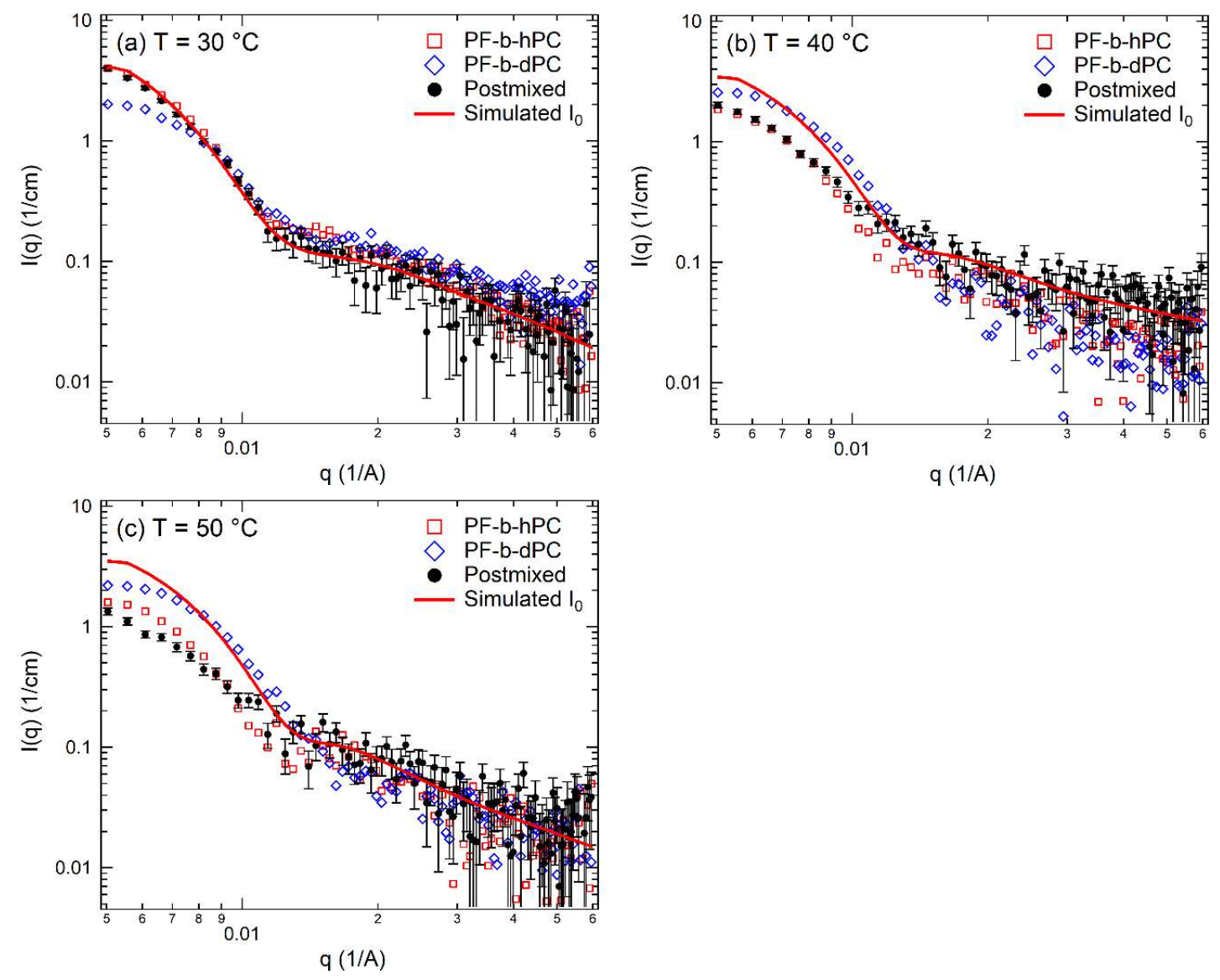

Figure S5. SANS profiles obtained from the isotopic mixture of PF- $b$-hPC and PF- $b-\mathrm{dPC}$ micelles (i.e., "post-mixed" solution) at 30,40 , and $50{ }^{\circ} \mathrm{C}$ for the first $5 \mathrm{~min}$ immediately after macroscopic mixing. The solid curves are the simulated SANS curves of the isotopic mixtures without chain exchanges using eq S8. 


\section{Shift Factors in Bulk and in Micellar Cores}

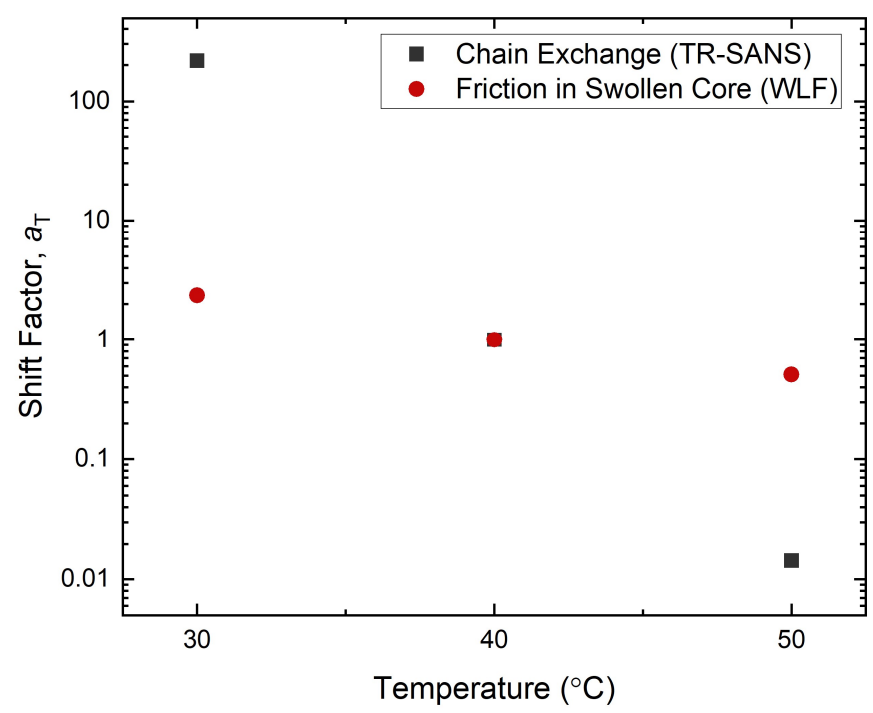

Figure S6. Temperature dependent shift factors for chain exchange kinetics (squares) and molecular friction of PF block in swollen core (dots) with the reference temperature of $40^{\circ} \mathrm{C}$. The latter was calculated using the WLF model and the $T_{\mathrm{g}}$ depression (eqs 4 and 5 in the main text, respectively). 


\section{Melt Viscosity Measurements}
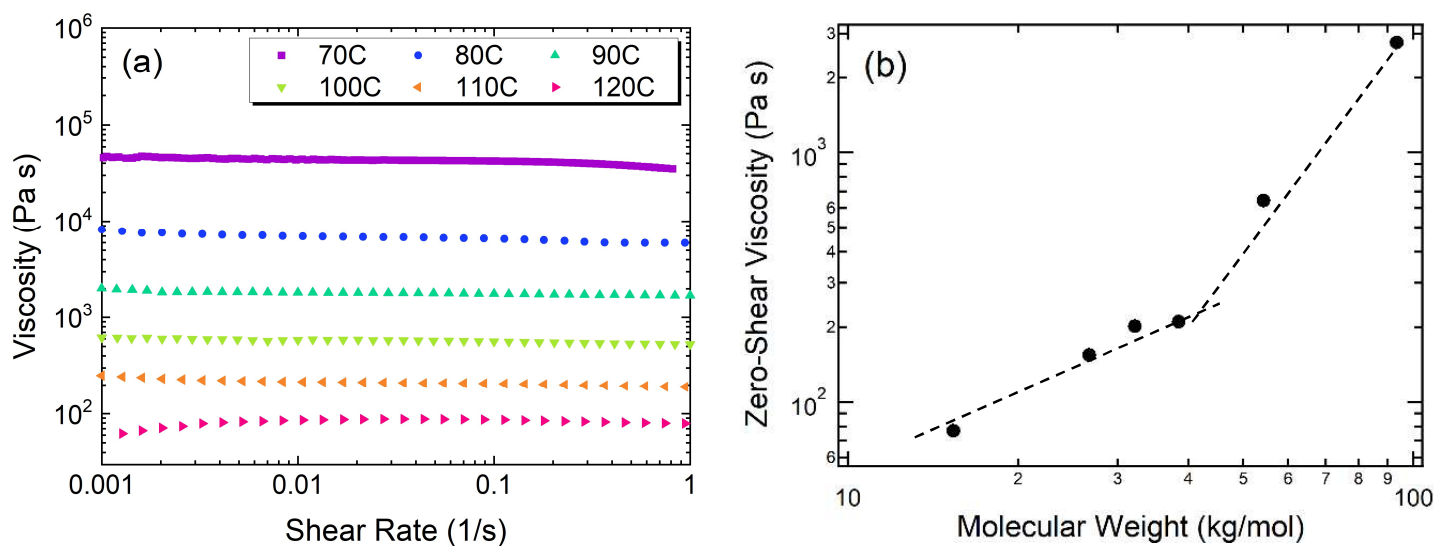

Figure S7. (a) Viscosity of PF homopolymer $(M=38 \mathrm{~kg} / \mathrm{mol})$ measured at 70, 80, 90, 100, 110 , and $120^{\circ} \mathrm{C}$. The zero-shear viscosity was defined from the extrapolation to zero shearrate. (b) Zero-shear viscosity $\left(\eta_{0}\right)$ at $110^{\circ} \mathrm{C}$ as a function of molecular weight of PF homopolymer. Dashed lines show the Rouse $\left(\eta_{0} \sim M^{1}\right)$ and the reptation $\left(\eta_{0} \sim M^{3.4}\right)$ scaling relations. 


\section{References for Supporting Information}

1. Radhakrishnan, K.; Switek, K. A.; Hillmyer, M. A., Synthesis of semifluorinated block copolymers by atom transfer radical polymerization. J. Polym. Sci. Pol. Chem. 2004, 42 (4), 853-861.

2. Valtola, L.; Hietala, S.; Tenhu, H.; Denifl, P.; Wilen, C.-E., Association behavior and properties of copolymers of perfluorooctyl ethyl methacrylate and eicosanyl methacrylate. Polymers for Advanced Technologies 2009, 20 (3), 225-234.

3. Marsat, J. N.; Heydenreich, M.; Kleinpeter, E.; Berlepsch, H. V.; Bottcher, C.; Laschewsky, A., Self-Assembly into Multicompartment Micelles and Selective Solubilization by Hydrophilic-Lipophilic-Fluorophilic Block Copolymers. Macromolecules 2011, 44 (7), 2092-2105.

4. Pedersen, J. S.; Svaneborg, C.; Almdal, K.; Hamley, I. W.; Young, R. N., A small-angle neutron and X-ray contrast variation scattering study of the structure of block copolymer micelles: Corona shape and excluded volume interactions. Macromolecules 2003, 36 (2), 416433.

5. Choi, S.-H.; Bates, F. S.; Lodge, T. P., Structure of Poly(styrene-b-ethylene-alypropylene) Biblock Copolymer Micelles in Squalane. J. Phys. Chem. B 2009, 113, 1384013848.

6. Choi, S.-H.; Bates, F. S.; Lodge, T. P., Molecular Exchange in Ordered Diblock Copolymer Micelles. Macromolecules 2011, 44 (9), 3594-3604.

7. Lund, R.; Willner, L.; Richter, D.; Dormidontova, E. E., Equilibrium Chain Exchange Kinetics of Diblock Copolymer Micelles: Tuning and Logarithmic Relaxation. Macromolecules 2006, 39 (13), 4566-4575. 